\title{
SEMBLANZA DE JOHN ERIC SIDNEY THOMPSON
}

(1898-1975)

\author{
Por Alberto Ruz LhuilLIER \\ Centro de Estudios Mayas \\ UNAM
}

Hace un par de meses, cuando esperaba respuesta de Eric Thompson a una carta mía del mes de julio en que le comentaba la llamada Segunda Mesa Redonda de Palenque, recibí unas líneas de Florence, su esposa, en que me informaba de su mal estado de salud, pese a lo cual ella esperaba que pronto se restablecería y dejaría el hospital para volver a casa. Desgraciadamente la siguiente noticia que tuve de él fue la nota en la prensa anunciando su fallecimiento.

Nuestro último encuentro fue hace dos años, en Saffron Walden. Pasé el día con Florence y Eric, visitamos la Universidad de Cambridge y algunas pequeñas iglesias de esa verde y suavemente ondulada campiña de Essex en que se había retirado. Nunca pensé entonces que no lo volvería a ver.

Quisiera, en esta breve nota, hacer resaltar lo que podría considerarse como un contraste entre el sabio y el hombre, pero que en realidad constituye la integración de una misma entidad, como las dos caras de una moneda.

\section{El sabio}

No creo que alguien pudiera negar que Eric Thompson fue el más sabio y el más brillante mayista, desde que se iniciara la investigación de la cultura maya hasta la actualidad. Ninguno de los investigadores que lo precedieron, a fines del siglo pasado y principios del nuestro, ni de los que fueron sus colegas de la Institución Carnegie de Washington o sus coetáneos durante medio siglo, ni de quienes entramos en el campo maya detrás de él, hace unos treinta años, ni por supuesto de las nuevas generaciones, ninguno se aproxima a lo que fue Eric Thompson. 
Un vistazo a su bibliografía nos ilustra sobre la intensidad de su dedicación, su entrega absoluta a la investigación, su constancia en la tarea que se había fijado, así como la extensa gama de sus preocupaciones científicas. Entre artículos, ensayos, reseñas, biografías, notas y libros, trabajos muy especializados o de amplia divulgación, son aproximadamente 200 títulos los que hemos contado.

En todos ellos resaltan sus cualidades de verdadero hombre de ciencia. Los temas que toca están tratados con toda seriedad; analiza los datos objetivamente, los clasifica, compara y valora dentro de un marco metodológico riguroso. Con aparente facilidad saca sus deducciones, en las que la imaginación no está excluida, pero en las que la fantasía no tiene cabida. Su síntesis es clara y coherente.

Para la presentación de sus investigaciones y conclusiones, Thompson contaba con magníficas dotes de escritor. Sus conceptos, perfectamente organizados, fluyen con nitidez, llevados por un lenguaje a la vez sencillo, preciso y rico, discretamente adornado con alusiones literarias o históricas en que aflora su amplia erudicción humanista.

Profesionalmente, fue mucho más que un arqueólogo. Las poblaciones mayances contemporáneas le interesaban tanto como los vestigios arqueológicos, y las lenguas, mitos y costumbres actuales eran tan importantes para él como los monumentos, la iconografía y los jeroglíficos prehispánicos. Participó en una decena de exploraciones y reconocimientos arqueológicos, principalmente en Belice, Yucatán y la costa guatemalteca del Pacífico. Realizó una investigación etnográfica entre grupos indígenas del Sur y Centro de Belice y recogió mitos modernos en distintas regiones del área maya.

Algunos temas le interesaron particularmente, cuyos estudios culminaron en importantes ensayos; recordaremos entre ellos el comercio, la navegación, el cultivo y uso del tabaco, el hule y el cacao. Pero lo que más profundamente lo cautivaba, y a lo que dedicó mayor preocupación, fue la epigrafía, el calendario y la religión, aspectos íntimamente ligados y que para él integraban un todo indivisible.

Desde el inicio de sus trabajos en el campo mayista, se enfrentó a los problemas de la escritura y la cronología. Le preocupaba que, disponiéndose de numerosas fechas mayas, no se pudiera con seguridad trasladarlas a nuestro calendario. La correlación establecida por Spinden era la que entonces más se aceptaba, aunque otra tenía también adeptos, elaborada por Goodman y ligeramente modificada por Martínez Hernández. Thompson afinó esta última, la que 
hasta la fecha es seguida por la mayor parte de los investigadores mayistas. Estudió las inscripciones jeroglíficas de numerosos sitios, y precisó fechas y cálculos calendáricos, la significación de algunos glifos lunares o de carácter esotérico, los signos que indican si debe contarse un intervalo de tiempo hacia adelante o hacia atrás; reconoció la existencia en el sistema calendárico de un ciclo de 819 días, probablemente asociado a la combinación de las influencias de siete deidades terrestres, nueve del inframundo y trece celestes. El resultado de años de intensa labor en este terreno se halla reunido en lo que considero la obra cumbre de Thompson: Maya Hieroglyphic Writing: an introduction, que completa su Catalog of Maya Hieroglyphs, y que pone al alcance del gran público la edición de bolsillo a la que dio el título de Maya hieroglyphs withouh tears. Su más reciente contribución en el terreno epigráfico y calendrárico es $A$ Commentary on the Dresden Codex, en que analiza este códice minuciosamente, página por página, renglón por renglón, figura por figura y glifo por glifo, calculando todo lo calculable, interpretando todo lo interpretable, sin dejar elemento matemático, cronológico, astronómico, esotérico, carente de explicación. La descripción de las deidades, las asociaciones glíficas y los probables o posibles augurios que de todo ello se desprenden constituyen una obra maestra de investigación que refleja la gran lucidez de su intelecto, el rigor científico con que trataba de resolver las interrogantes que plantea una escritura sólo parcialmente descifrada, su privilegiada memoria y su coherencia en la síntesis.

Alguna vez me dijeron que Eric Thompson era pastor anglicano. En 25 años de trato, nunca manifestó en lo más mínimo una actitud que pudiera interpretarse como la de un ministro religioso; en realidad, nada que demostrara religiosidad o, por el contrario, anti-religiosidad. Pero creo que su enfoque de la cultura y de la historia de los mayas se debía a un espíritu profundamente religioso. Dedicó algunos de sus trabajos a cuestiones específicas relacionadas con conceptos esotéricos que acompañan el calendario o aparecen en los textos jeroglíficos: las deidades nocturnas de la Serie Suplementaria; la cuadruplicidad que abarca dioses, elementos naturales, colores y puntos cardinales; los símbolos acuáticos, la diosa lunar y sus conexiones; las deidades extrañas a la religión maya que introdujeron invasores; ciertos ritos particulares; numerosos mitos aún vigentes. En su última gran obra de divulgación, Maya History and Religion, dedica cerca de 250 páginas a deidades, creencias y prácticas religiosas. 
Para Thompson, fueron valores espirituales -el carácter propio de los mayas, moderado en todo y esencialmente pacífico y su devoción religiosa - los que sirvieron de motor a la civilización maya, los que explican su nacimiento y desarrollo y los que le dieron su brillantez. Y fueron también causas espirituales - la intrusión de ideas exóticas, creencias religiosas y ritos menos humanos que los propiamente mayas, "influencias de una cultura moralmente más débil" - la "polilla" procedente del centro de México, las que provocaron su desintegración, su degeneración y el colapso final.

Su actitud espiritualista lo condujo, no sólo a compartir con Spinden y Morley la idea de que las inscripciones mayas carecían por completo de contenido histórico, sino a elaborar lo que llamó "la filosofía del tiempo", sosteniendo que los monumentos mayas con inscripciones, más de un millar conocidos, "tratan exclusivamente del paso del tiempo, de datos sobre la luna y el planeta Venus, de cálculos calendáricos y de asuntos sobre los dioses y los rituales implícitos en estos temas".

Sin embargo, después de conocer los trabajos de Berlin, Proskouriakoff, Kelley, en que se interpretan las inscripciones como sintéticas informaciones de carácter histórico, no se aferró a su convicción, como lo hizo Morley con su teoría del Viejo y del Nuevo Imperio, sino que investigó con este nuevo enfoque y ofreció datos que contradicen diametralmente lo que había sostenido hasta entonces, relacionando los glifos calendáricos Cipactli de una estela de Seibal con el nombre de la familia que gobernaba en Potonchan en el momento de la Conquista, reconociendo así la presencia de nombres de personajes históricos en las inscripciones mayas. $\mathrm{Y}$ es que Eric Thompson era de una honradez científica a toda prueba.

Al poco tiempo de iniciarse en los estudios mayistas, Thompson sintió la necesidad de difundir los conocimientos que iba adquiriendo, y esta actitud la conservó a lo largo de toda su fructífera vida de investigador. En 1931 escribió con Gann The History of the Maya, y sucesivamente, con conocimientos cada vez más amplios, profundos y precisos, puso al alcance del público general excelentes síntesis históricas y culturales como México before Cortés (1933), The Rise and Fall of Maya Civilization (1954), Maya History and Religion (1970). Su Maya Archaeologist (1963) es más que el simple relato de sus experiencias y aventuras como arqueólogo, ya que presenta importantes datos sobre los sitios que exploró, en Yucatán, Belice, El Petén y Copán, sin que ello justifique el cambio dado 


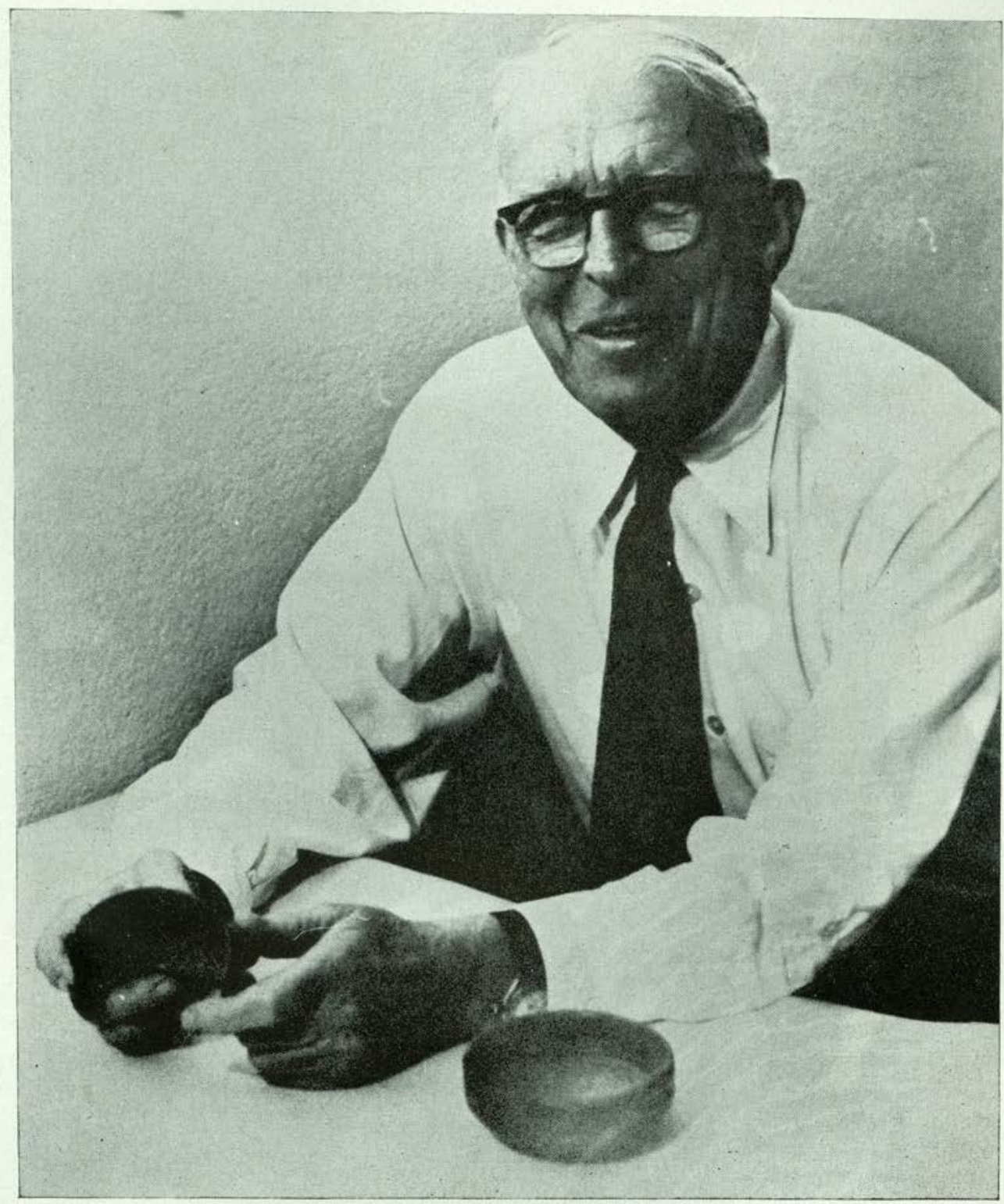

John Eric Sidney Thompson.

Estudios de Cultura Maya. Vol. X, 1976/7

Instituto de Investigaciones Filológicas/ Facultad de Filosofía y Letras Centro de Estudios Mayas, UNAM 
al título en la edición mexicana (Arqueología Maya, en vez de Arqueólogo Maya).

En síntesis, puede afirmarse que Eric Thompson fue el más brillante de los sabios mayistas, por su intensa, constante, profunda y rigurosa labor científica, por la gran diversidad de los temas que investigó, por sus valiosas aportaciones sobre cuestiones muy especializadas y por la amplísima difusión que dio a la historia y a la cultura de los mayas.

\section{El hombre}

Brillante como hombre de ciencia, Thompson en lo personal fue un hombre modesto. En ningún momento, ni en su obra ni en su vida trató de presentarse como el ser excepcionalmente dotado que en realidad era. No encontramos, en sus libros, en su correspondencia, en el recuerdo de su trato directo, algo que implicara no digamos vanidad, sino orgullo, que hubiera sido bastante legítimo dada su personalidad científica.

Fue hombre sencillo, discreto, y casi me atrevería a decir tímido. Sencilla fue su vida, dedicada al estudio, la investigación, el pensar creativo, la tarea literaria de ofrecer al mundo el resultado de sus meditaciones. De regreso a Inglaterra, después de muchos años de vivir en los Estados Unidos, trabajando como miembro del Departamento de Historia de la Institución Carnegie, se encerró en una modesta y agradable casita, y lo que se supo de él fueron sus publicaciones, que una tras otra, casi sin interrupción iban siguiéndose. Completaban su vida el cuidado afectuoso con que rodeaba a Florence, su enfermiza esposa, y la atención a los rosales de su jardín. Poca gente, en Saffron Walden y sus alrededores, al parecer en la Universidad vecina de Cambridge, y en las esferas oficiales del Reino Británico sabía que en aquel rincón de Essex vivía y trabajaba infatigablemente el más sabio de los investigadores del pueblo maya.

Sus colegas y amigos sí sabíamos más de él. Lo poníamos al tanto de nuestros trabajos, de campo o de gabinete; le hacíamos consultas, le exponíamos nuestras dudas o le manifestábamos nuestra ignorancia. No había carta que no contestara en breve plazo, labor que no despertara su interés, dudas que no tratara de resolver, lagunas o fallas nuestras que no llenara o corrigiera con su sabiduría y con amistoso tacto.

Su discreción, la aplicaba a sí mismo cuando, sin el menor alarde, presentaba nuevas ideas, los complejos cálculos calendáricos de un 
monumento, la significación de un conjunto jeroglífico, o cuardo titulaba su obra mayor sobre la escritura jeroglífica maya: "una introducción", en realidad 350 páginas de densa sabiduría. La aplicaba también cuando opinaba de trabajos ajenos con los que no estaba de acuerdo, e incluso cuando se trataba de audacias de charlatanes.

En estos casos, más que una justificada reprobación tajante era una burla irónica la que usaba para poner al responsable en el lugar merecido.

No soy psicólogo e ignoro si existe un lindero bien definido entre la modestia y la timidez, pero creo que Thompson, brillante sabio, era al mismo tiempo un hombre tímido. Ello explicaría, a mi juicio, su resistencia a presentarse en público, como conferencista o profesor. Con la amplitud y profundidad de sus conocimientos, su capacidad para organizar y sintentizar su pensamiento, su excelente manejo del idioma, incluso del español, no hubiera tenido la menor dificultad en cautivar a cualquier auditorio, culto o profano. Y sin embargo, muy pocas fueron las conferencias que sustentó, y al parecer nunca aceptó dictar curso alguno. Esto no puede atribuirse a una actitud egoísta de negarse a transmitir sus conocimientos, ya que sus numerosos y magníficos libros de difusión demuestran todo lo contrario. Una excepción en su actitud fue la de dirigir a un grupo de turistas ingleses, a principios de 1973, pero no dejó de burlarse de su tarea como guía cuando me escribió que estaría en México "muy ocupado, diciendo cuántas vírgenes fueron arrojadas al cenote y demostrando cómo los Mayas abrían el pecho de las víctimas para sacarles el corazón".

Parece que fue hasta casi mediados de 1973 cuando las autoridades inglesas lo descubrieron. El discreto sabio mayista y su esposa fueron entonces invitados al banquete dado en el Castillo de Windsor en honor del Presidente de México y de la señora de Echeverría; Thompson habló unos minutos con la Reina y le contó al Presidente que había pasado su luna de miel en las ruinas de Cobá. Me es difícil imaginar a Thompson en medio de la pomposa ceremonia, como tampoco me lo imagino, pocos meses después, en el acto de recibir un doctorado honorífico en la Universidad de Cambridge en otra "big ceremony" como me lo escribió, disfrazando su modestia —o timidez - bajo el comentario jocoso: "Tendré que llevar una toga escarlata".

Sus cartas de los últimos años mostraban preocupaciones por su estado de salud, preocupaciones que disfrazaba también con la broma: "un abrazo afectuoso aunque algo débil del viejo Eric" o "un fuer- 
te abrazo del viejito estropeado", eran sus palabras de despedida, en español o en maya ("un abrazo del noh xib"). Y al enviarme para nuestros Estudios de Cultura Maya, el artículo sobre nombres calendáricos entre los mayas chontales que publicamos en este volumen, al que calificó como "not very exciting", consideraba que "probablemente será la última cosa que escriba, pues decidí retirarme y no escribir más sobre los mayas". Pero el humor, de nuevo, suavizaba y disimulaba el pesimismo del anuncio: "Espero no ser uno de estos cantantes de ópera que siempre están retirándose, pero siempre regresando". Sin embargo, su espíritu de investigador seguía intacto, y añadía: "Espero escribir un estudio sobre los marinos de John Hawkins capturados en 1580 en Veracruz y Tampico y llevados más tarde ante la Inquisición".

Sobre los mayas, decía ya haberlo escrito todo. Manifestaba cansancio y también desagrado respecto a las afirmaciones rotundas, espectaculares y atrevidas por falta de justificación seria, que algunos mayistas, novatos generalmente, lanzaron en los últimos tiempos, y respecto de las cuales hacía chistes que apenas ocultaban la molestia que le producían tales afirmaciones, reproducidas en la prensa y aun en publicaciones que pretenden ser científicas; "Estoy feliz de estar ahora retirado de este campo", me escribió en su última carta, después de aludir concretamente a interpretaciones fantasiosas de las inscripciones de Palenque. A los responsables de estas fantasías se refirió cuando, al leerle Florence en el hospital una nota mía que sobre el mismo tema publicó la Dirección General de Difusión en IMAGEN, periódico mural de nuestra Universidad, sólo comentó: "these young people do have wild ideas" (estos jóvenes tienen de veras ideas descabelladas). Son las últimas palabras suyas sobre la investigación mayista que, indirectamente, me llegaron.

Terminaré con otras palabras de Eric Thompson, escritas a principios de este año, en respuesta al cable de felicitación que le envié con motivo de haber sido designado Caballero por la Reina de Inglaterra. No hay en ellas ninguna expresión de vanidad satisfecha, y por el contrario, a través del humor con que él mismo se hace objeto de burla, trasciende su modestia, se oculta la timidez con que debió verse convertido en Sir Eric Thompson: "Es quizás una buena cosa que el Imperio Británico, como el Imperio Mexicano de Maximiliano, ya no exista. Seis nuevos Caballeros del Imperio Británico fueron creados el día de Año Nuevo. De éstos, P. G. Wodehous, el novelista, tiene 91 años (murió uno o dos meses después); Charlie Chaplin tiene $85 \mathrm{y}$ anda en silla de ruedas; yo tengo 76 . 
Si nos llamaran a defender el honor del Imperio y sus empresas en una justa o torneo, me temo que no tendríamos mucho éxito".

Sólo unos meses iba a vivir el nuevo Caballero del Imperio Británico, pero el caballero del espíritu, el dignatario de la única orden que realmente implica distinción merecida, la de la sabiduría, el más distinguido científico mayista que fue J. Eric S. Thompson, estará siempre vivo en el recuerdo agradecido de quienes aprendimos mucho de él, personalmente y en sus obras, y su nombre, sin necesidad de título nobiliario, permanecerá eternamente ligado al conocimiento de la historia y de la cultura de los mayas.

Octubre 1975.

\section{BIBLIOGRAFIA RECOPILADA POR ENRIQUE VILORIA V.}

1925 "The meaning of the maya months". MAN, 25: 121-123. London.

1926 "Chichen Itza, a centre of the maya civilization". The Field. London.

1927 "A correlation of the mayan and european calendars". Field Museum of Natural History, Anthropological Series, 17 (1): 1 22. Chicago.

1927a The civilization of the mayas. Field Museum of Natural History, Leaflet 25, Chicago. (2a. edición en 1932, 3a. en 1936, 5a. en 1953, 6a. en 1958).

$1927 \mathrm{~b}$ (con T. A. Joyce y J. C. Clark) "Report en the British Museum expedition to British Honduras, 1927". Journal Royal Anthropological Institute, 57: 295-323.

1927 c "The elephant heads in the Waldeck manuscripts". Scientific Monthly, 25: 392-398. Lancaster.

1928 "The 'children of the sun' and Central America". Antiquity, 2: 161167. Hope, New Jersey.

1928a "Some new dates from Pusilha". MAN, 28 (7): 95-97. London.

1929 "Back with relics of ancient mayas". (Eric Thompson reports to Field Museum on explorations in British Honduras) The New York Times, 28 de julio de 1929. New York.

1929a "Comunicaciones y comercio de los antiguos mayas". Anales de La Sociedad de Geografia e Historia de Guatemala, 6 (1): 40-44. Guatemala.

1929b "Maya chronology: glyph G of the lunar series". American Anthropologist, 31 (2): 223-231. Menasha.

1930 "An ancient delicacy" Field Museum News, 1 (7): 3. Chicago. (Reproducido con título de "mayas bred dogs for sacrifice" en El Palacio, 28: 101. Santa Fé, Calif.).

1930 a "The causeways of the Coba district, eastern Yucatan". Congreso Internacional de Americanistas, 23, New York, 1928. p. 181-184. New York.

1930b Domesticated fauna of ancient Middle America (Ms.), Chicago.

$1930 \mathrm{c}$ Ethnology of the mayas of southern and central British Honduras. 
Field Museum of Natural History, Anthropological Series, 17 (2). Chicago.

1931 Achaeological investigations in the soutern Cayo distric British Honduras. Field Museum of Natural History, Anthropological Series, 17 (3). Chicago.

1931a "Copan, an ancient maya metropolis". The Open Court, 45: 737-753. Chicago.

1931b (con Thomas W. F. Gann) The history of the maya, from the earliest time to the present day. London-New York.

1931c "Models of zapotec temple and maya pyramid". Field Museum News, 2 (3). Chicago.

1932 (con H. E. Pollock y J. Charlot) A preliminary study of the ruins of Coba, Quintana Roo, Mexico. C.I.W.,* Pub. 424. Washington.

1932a "The humming bird and the flower". Maya Society Quarterly. 1: 120-122. Baltimore.

1932b "A maya calendar from the Alta Vera-Paz, Guatemala". American Anthropologist, 34 (3): 449-454. Menasha.

1932c "Mexican household gods". Field Museum News, 3 (5). Chicago. 1932d The solar year of the mayas at Quirigua, Guatemala. Field Museum of Natural History, Anthropological Series. 17 (4). Chicago.

$1932 \mathrm{e}$ "Some jade-inlaid teeth of ancient mayas". Field Museum News, (3): 3. Chicago.

1933 An ancient peruvian gold beaker. Field Museum News, 4 (9). Chicago. 1933a "Aztec and maya lords of the night". Field Museum of Natural history, Anthropological Series. 17 (4): 414-4-18. Chicago.

1933b (con Sylvanus G. Morley) "Hieroglyphic research". C.I.W. Year Book, 32: 97-98. Washington.

1933c "Aztec priesthood, aports and writings". Mexican Life, 9 (10): 15-16 y 48-58. México.

1933d Mexico before Cortez. New York-London.

1934 "Excavations in British Honduras". C.I.W. Year Book, 33: 95-98. Washington.

1934a "Maya chronology: the fifteen tun glyph". C.I.W., Pub. 436, Contrib. 11: 245-254. Washington.

1934b "Mexican stone sculpture". Field Museum News, 5 (11). Chicago.

$1934 \mathrm{c}$ "Sky bearers, colors and directions in maya and mexican religion". C.I.W., Pub 436, Contrib. 10: 209-242. Washington.

1935 An ancient peruvian quipu. Field Museum News, 6 (3). Chicago

1935a "The dates on altar U, Copan". Maya Research, 2 (1): 11-13. New Orleans.

1935b Lacquered vessels from Peru. Field Museum News, 6 (1). Chicago. 1935c The tibetan god of death. Field Museum News, 6 (2). Chicago.

1936 Archaeology of South America. Field Museum of Natural History, Anthropological Leaflet, 33. Chicago.

1936a La civilización de los mayas. Tr. de The civilization of the mayas por Samuel Ramos. Pub. del Depto. de Bibliotecas de la Secretaría de Educación Pública. México.

* Carnegie Institution of Washington. 
1936b "The dates of the Temple of the Cross, palenque". Maya Research, $3(3-4)$ : 287-293. New Orleans.

1936c "Exploration in Campeche and Quintana Roo and excavations at San Jose, British Honduras". C.I.W., Year Book, 35: 125-128. Washington.

1936d "An eccentric flint from Quintana Roo, Mexico". Maya Research, 3 (3-4): 316-318. New Orleans.

1936e "Lunar inscriptions in the Usumacinta Velley". El México Antiguo, 4 (4): 69-72. México.

$1936 \mathrm{f}$ "A note on Scherzer's visit to Quirigua". Maya Research, 3 (3-4): 330-331. New Orleans.

1937 (con Lawrence Roys y R. C. Long) "Maya chronology; the correlation question". C.I.W., Pub. 456, Contrib. 14: 51-104. Washington.

1937a "A new method of deciphering yucatecan dates with special reference to Chichen Itza". C.I.W., Pub. 483, Contrib. 22. Washington.

1938 (con Alfred Vincent Kidder) "The correlation of maya and christian chronologies". C.I.W., Pub. 501: 493-510. Washington.

1938a Introd. a la obra: The high priest's grave. Chichen Itza, Yucatan, Mexico. de: Edward H. Thompson. Field Museum of National History, Anthropological Series, 27 (1). Chicago.

1938b "Reconnaissance and excavation in British Honduras". G.I.W., Year Book, 37: 16 17. Washington.

1938 c "Sixteenth and seventeenth century reports on the chol mayas". American Anthropologist, 40: 584-603. Menasha.

1939 Excavations at San Jose, British Honduras. C.I.W., Pub. 506. Washington.

1939a (editor con Francis B. Richardson) Traducción hecha bajo la supervisión de Charles P. Bowditch de "Gesammelte Abhanlungen zur Amerikanischen Sprach-und Alterthumskund" Vols. I-V, de Eduard Seler, Berlín 1902-1923.

$1939 \mathrm{~b}$ "The Moon goddess in Middle America: with notes on related deities". C.I.W., Pub. 509, Contrib. 29: 123-173. Washington.

1940 "Apuntes sobre la estela número 5 de Balakbal, Q. R.". Revista Mexicana de Estudios Antropológicos, 4: 5-9. México.

1940a "Bibliografías de antropólogos, Thomas William Francis Gann, 1867. 1938". Boletín Bibliográfico de Antropología Americana, 4: 158-163. México.

1941 "Apuntes sobre las supersticiones de los mayas de Socotz, Honduras Británica". Los mayas antiguos, 101-110. México.

1941a "A coordination of the history of Chichen Itza with ceramic secuences in Central America". Revista Mexicana de Estudios Antropológicos, 5 (2-3): 97-111. México.

$1941 b$ "Dating of certain inscription of non-maya origin". C.I.W., Div. of Hist. Res.,* Theoretical Approaches to Problems, 1. Cambridge.

1941c "The missing illustrations of the Pomar Relacion". C.I.W., Div. of Hist. Res., Notes on Middle American Archaeology and Ethnology, 1 (4): 15-21. Cambridge.

* Division of Historical Research. 
1941d "The prototype of the Mexican Codices Telleriano Remensis and Vaticanus A". C.I.W., Div. of Hist. Res., Notes on Middle American Archaeology and Ethnology, 1 (6): 24-26. Cambridge.

1941 e "Las relaciones entre Tula y las culturas de Cholula, Oaxaca, Yucatán y Centroamérica". Sociedad Mexicana de Antropología, Boletín, 2. México.

$1941 \mathrm{f}$ "Yokes or ball game belts?". American Antiquity, 6 (4): 320-326. Salt Lake City.

1942 "Guatemala Pacific Coast". C.I.W., Year Book, 41; 267-269. Washington.

1942a "Las llamadas fachadas de Quetzalcouatl". Congreso Internacional de Americanistas, 27, México, 1939. Actas 1: 391-400. México.

1942b "Maya arithmetic". C.I.W., Pub. 528, Contrib. 36: 37-62. Washington.

1942c "Observations on glyph G of the lunar series". C.I.W., Div. of Hist. Res., Notes on Middle American Archaeology and Ethnology, 1 (7): 27-29. Cambridge.

1942d "Late ceramic borizons at Benque Viejo, Honduras". C.I.W., Pub. 528, Contrib. 35: 1-35. Washington.

$1942 \mathrm{e}$ "Representations of Tezcatlipoca at Chichen Itza". C.I.W., Div. of Hist. Res., Notes on Middle American Archaeology and Ethno$\log y, 1$ (12): 48-50. Cambridge.

1943 "A figurine whistle representing a ball-game player". C.I.W., Div. of Hist. Res., Notes on Middle American Archaeology and Ethno$\log y, 1$ (25): 160-162. Cambridge.

1943a "The initial series of stela 14, Piedras Negras, Guatemala, and a date on stela 19, Naranjo, Guatemala". C.I.W., Div. of Hist. Res., Notes on Middle American Archaeology and Ethnology, 1 (18): 113-116. Cambridge.

1943b "Maya epigraphy: a cycle of 819 days". C.I.W., Div. of Hist. Res., Notes on Middle American Archaeology and Ethnology, 1 (22): 137-151. Cambridge.

1943c "Maya epigraphy: directional glyphs in counting". C.I.W., Div. of Hist. Res., Notes on Middle American Archaeology and Ethno$\log y, 1$ (20): 122-126. Cambridge.

1943d "Pitfalls and stimuli in the interpretation of history through loan words". Tulane University, Middle american Research Institute, Philological and Documentary Studies, 1 (2): 17-28. New Orleans.

$1943 \mathrm{e}$ "Representations of Tlalchitonatiuh at Chichen Itza, Yucatan, and El Baul, Escuintla". C.I.W., Div. of Hist. Res., Notes on Middle American Archaeology and Ethnology, 1 (19): 117-121. Cambridge.

$1943 \mathrm{f}$ "Some sculptures from southeastern Quezaltenango". C.I.W., Div. of Hist. Res., Notes on Middle American Archaeology and Ethnology, 1 (17): 100-112. Cambridge.

$1943 \mathrm{~g}$ "A trial survey of the southern maya area". American Antiquity, 9 (1): 106-134. Salt Lake City.

1944 "La correlación más probable entre las cronologías maya y cristiana". Diario de Yucatán, 9 de febrero, Mérida, Yucat'n, México.

1944a "The dating of seven monuments at Piedras Negras". C.I.W., Div. 
of Hist. Res., Notes on Middle American Archaeology and Ethno. logy, 2 (39): 65-82. Cambridge.

1944b "The fish as a maya symbol for counting and further discussion of directional glyphs". C.I.W., Div. of Hist. Res., Theoretical Approaches to Problems, 2. Cambridge.

1944c "Hieroglyphic research". C.I.W., Year Book, 43: 172-173. Washington.

1944d "Jottings on inscriptions at Copan". C.I.W., Div. of Hist. Res., Notes on Middle American Archaeology and Ethnology, 2 (38): 48-64. Cambridge.

1944e (con Sylvanus G. Morley) "Reconnaissance in Mexico and Guatemala". C.I.W., Year Book, 43: 169-172. Washington.

$1944 \mathrm{f}$ "Variant methods of date recordings in the Jatate drainage, Chiapas". C.I.W., Div. of Hist. Res., Notes on Middle American Archaeology and Ethnology, 2 (45): 133-138. Cambridge.

1945 "Escritura jeroglífica. Aritmética y astronomía de los mayas". Enciclopedia Yucatanense, 2: 309-342. México.

1945a "Hieroglyphic Research". C.I.W., Year Book, 44: 172-173. Washington.

1945b "The inscription on the altar of zoomorph O, Quirigua". C.I.W., Div. of Hist. Res., Notes on Middle American Archaeology and ethnology, 2 (56): 189-199. Cambridge.

1945c "A survey of the northern maya area". American Antiquity, 11 (1): 2-24. Salt Lake City.

1945d "Un vistazo a las 'ciudades' mayas: su aspecto y su función". Cuadernos Americanos, 4 (2): 136-149. México.

1946 (con Alfonso Caso et al) “ ¿Conocieron la rueda los indígenas meso-

(') americanos?". Cuadernos Americanos, 5 (1): 193-207. México.

$1946 \mathrm{a}$ "The dating of structure 44, Yaxchilan, and its bearing on the sequence of texts at that site". C.I.W., Div. of Hist. Res., Notes on Middle American Archaeology and Ethnology, 3 (71): 62-74. Cambridge.

1946b "Hieroglyphic research". C.I.W., Year Book, 45: 208-209. Washington.

1946c "Sobre la Cuarta Mesa Redonda". El Palacio, 53: 355-356. Santa Fe, California.

1946d "Some uses of tabacco among the maya". C.I.W., Div. of Hist. Res., Notes on Middle American Archaeology and Ethnology, 3 (61): 1-6. Cambridge.

1946e "Tattooing and scarification among the maya". C.I.W., Div. of Hist. Res., Notes on Middle American Archaeology and Ethnology, 3 (63): 18-25, Cambridge.

1947 "Hieroglyphic research". C.I.W., Year Book, 46: 188-189. Washington.

1947a (con Tatiana Proskouriakoff) "Maya calendar round dates such as 9 ahau 17 mol". C.I.W., Div. of Hist. Res., Notes on Middle American Archaeology and Ethnology, 3 (79): 143-150. Cambridge.

1948 "An archaeological reconnaissance in the Cotzumalhuapa region, Es- 
cuintla, Guatemala". C.I.W., Pub. 574, Contrib. 44: 1-94. Washington.

1949 (con Ignacio Bernal) "Murciélagos". Tlalocan, 3: 95 y 191. México.

1949 a "Tentativa de reconocimiento en el area maya meridional". Antropología e Historia de Guatemala, 1 (2): 23-48. Guatemala. 2a. Ed. 1957.

1950 Maya hieroglyphic writing; an introduction. C.I.W., Pub. 589. Washington. (2a. Ed. Norman, 1960).

1950a Rubber in America before 1492. Boston, Godfrey L. Cabot.

1950b "Some anecdotes about Vay". Morleyana, 252-256. Santa Fe, California.

1951 "Bibliografía de Thomas A. Joyce". Boletín Bibliográfico de Antropología Americana, 13 (2): 252-258. México.

1951a "Canoes and navigation of the maya and their neighbors". Journal Royal Anthropological Institute, 79: 69-78. London.

1951b "Hieroglyphic research". C.I.W., Year Book, 50: 244-245. Washington.

1951c "The Itza of Tayasal, Peten". Homenaje al Dr. Alfonso Caso, p. 389-400. México.

1952 "Aquatic symbols common to various centers of the classic period in Mesoamerica". Acculturation in the Americas. Proceedings and Selected Papers of the XXIX International Congress of Americanist, New York, 1949. 1: 31-36. Chicago.

1952a "La inscripción jeroglífica del tablero del Palacio, Palenque". Anales del Instituto Nacional de Antropología e Historia, 4: 61-68. México.

1952b "The introduction of Puuc style of dating at Yaxchilan". C.I.W., Dep. of Archaeology, Notes on Middle American Archaeology and Ethnology, 4 (110): 196-202. Cambridge.

1952 c "Waxen idols and a sacrifical rite of the lacandon". C.I.W., Dep. of Archaeology, Notes on Middle American Archaeology and Ethnology, 4 (109): 193-196. Cambridge.

1953 "The character of the maya". Congreso Internacional de Americanistas, 30, Cambridge, 1952, p. 36-40. London.

1953a "Hieroglyphic research". C.I.W., Year Book, 52: 287-288. Cambridge.

1953b "Relaciones entre Veracruz y la región maya". Revista Mexicana de Estudios Antropológicos, 13 (2-3): 447-454. México.

1953c "A stela at San Lorenzo, southeastern Campeche". C.I.W., Dep, of Archaeology, Notes on Middle American Archaeology and Ethno$\log y, 4$ (115): 228-231. Cambridge.

1954 "El área maya norte". Yan, 1 (3): 3-35. México.

1954a "Excavations of residential-type structures at Mayapan". C.I.W., Year Book, 53: 279-281. Washington.

1954b "Memoranda on some dates at Palenque, Chiapas". C.I.W., Div. of Hist. Res., Notes on Middle American Archaeology and Ethno$\log y, 5$ (120): 45-52. Cambridge.

1954 c "A presumed residence of the nobility at Mayapan". C.I.W., Current Reports, 2 (19): 71-89. Cambridge. 
1954d The rise and fall of maya civilization. Norman, University of Oklahoma Press.

1955 Introducción a la obra: Ancient maya paintings of Bonampak, México. C.I.W., Supplementary Publications, 46. Washington.

1955a (con Karl Ruppert y Tatiana Proskouriakoff) Bonampak, Chiapas, México. C.I.W., Pub. 602. Washington.

1955b (con Donald E. Thompson) "Excavations of a residential-type group in Mayapan". C.I.W., Year Book, 54: 273-275. Washington.

1955 c "Mayapan, última etapa de una civilización moribunda: la maya". Congreso Internacional de Americanistas, 31, Sao Paulo, 1954. Actas, p. 667-672. Sao Paulo, Brasil.

1955d (con Donald E. Thompson) "A noble's residence and its dependencies at Mayapan". C.I.W., Dep. of Archaeology, Current Reports, 2 (25): 225-252. Cambridge.

1956 "Chronological decipherments from Uaxactun, Naranjo and Ixlu Peten". C.I.W., Dep. of Archaeology, Notes on Middle American Archaeology and Ethnology, 5 (127): 89-94. Cambridge.

1956a "Notes on the use of cacao in Middle America". C.I.W., Dep. of Archaeology, Notes on Middle American Archaeology and Ethno$\log y, 5$ (128): 95-116. Cambridge.

1957 "Deities portrayed on censers at Mayapan". C.I.W., Dep. of Archaeology, Current Reports, 2 (40): 599-632. Cambridge.

1958 "Hieroglyphic research". C.I.W., Year Book, 56: 412. Washington.

1958a Introd. a la obra: Thomas Gage's travels in the new world. Norman, University of Oklahoma Press.

1958b "Research in maya hieroglyphic writing". Unión Panamerican, Social Science Monographs, 5: 43-52. Washington. Tr. al español en 1960. en Unión Panamericana, Estudios Monográficos, 5: 38-53. Washington.

1958c "Symbols, glyphs and adivinatory almanacs for diseases in the maya Dresden and Madrid codices". American Antiquity, 23: 297-308. Salt Lake City.

1959 "La contribución yucateca a los estudios mayistas". El Nacional, 22 de marzo de 1959. México.

1959a Grandeza y decadencia de los mayas. Tr. de Lauro José Zavala. México, Fondo de Cultura Económica.

1959b "Realizaciones intelectuales y artísticos de los mayas". Revista de la Universidad de Yucatán, marzo abril, p. 26-75. México.

1959 c "The role of caves in maya culture". Museum fur Volkerkunde in Hamburg, Mitteilungen, 25: 122-129. Hamburg.

1959d "Systems of hieroglyphic writing in Middle America and methods of decipherment them". American Antiquiti, 24 (1): 349-364. Salt Lake City. También en Ancient Mesoamerica, Graham Ed., Berkeley, 1960.

1960 "Maya warfare, art and architecture". Mexican Life, 36 (7) : 22-24. México.

1961 "A blood-drawing ceremony painted on a maya vase". Estudios de Cultura Maya, 1: 12-20. México. 
1961a "John Hawkins ship's boys in Mexico". Homenaje al Dr. Pablo Martínez del Río, p. 495-504. México.

1961b "Notes on a plumbate vessel with shell inlay and on chiclero's ulcer". Essays in pre-colubian art and archaeology, Cambridge, Harvard University Press, p. 171-175.

1962 A catalog of maya hieroglyphs. Norman, University of Oklahoma Press.

1962a "Convocation address". Expedition, 4 (3): 14-16. Philadelphia.

$1962 \mathrm{~b}$ "El misterio del diccionario maya de Solana". Estudios de Cultura Maya, 2: 11-16. México.

1963 "Algunas consideraciones respecto al desciframiento de los jeroglíficos mayas". Estudios de Cultura Maya, 3: 119-148. México.

1963a Maya archaeologist. London, R. Hale Limited.

1963b "Pictorial synonyms and homonyms in the maya Dresden Codex". Tlalocan, 4 (2): 148-156. México.

1964 "1914: La Carnegie Institution of Washington ingresa al campo maya". Estudios de Cultura Maya, 4: 167-176. México.

1964 a "Trade relations between the maya highlands and lowlands". Congreso Internacional de Americanistas, 35, México, 1962. Actas y Memorias, 1: 245-247. México. (original en: Estudios de Cultura Maya, 4: 13-50. México).

1965 "Archaeological synthesis of the southern maya lowlands". Handbook of Middle American Indians, 2: 331-359. Austin.

1965a "To Coba on the great stone road". en: They found buried cities, Wauchope, Ed. p. 332-352. Chicago.

1965b "A cooper ornament and a stone mask from Middle America". American Antiquity, 30 (3): 343-345. Salt Lake City.

1965 c "Maya creation myths" (part I) Estudios de Cultura Maya, 5: 1332. México.

1965d "Maya hieroglyphic writing". Handbook of Middle American Indians, 3: 632-658. Austin.

1965 e Preliminary decipherments of maya glyphs I. Saffron Walden: England.

$1965 \mathrm{f}$ "The Relation of Tecuanapa, Guerrero". Tlalocan, 1: 85-96. México.

1966 "Ayopechtli, an aspect of the nahua goddess of the maguey". Congreso Internacional de Americanistas, 36, Barcelona-Sevilla, 1964. 2: 103-106. Sevilla, España.

1966a "The maya central area at the spanish conquest and later: a problem in demography". Proceedings of the Royal Anthropological Institute of Great Britain and Ireland, p. 23-37. London.

1966b "Maya hieroglyphs of the bat as methaphorgrams". MAN, 1 (2): 174-184. London.

1966c "Merchand gods of Middle America". Summa antropológica en homenaje a Roberto J. Weitlaner, p. 159-172. México.

1966d Preliminary decipherments of maya glyphs 2-3. Saffron Walden, England.

1967 Introd. a la obra: Ancient maya relief sculpture: rubings by Merle Greene. New York, Museum of Primitive Art. 
1967a Introd. a la obra: The book of Chilam Balam of Chumayel. by Ralph L. Roys. Norman, University of Oklahoma Press.

1967b "Maya creation myths" (part II). Estudios de Cultura Maya, 6: 1543. México.

1967 c Preliminary decipherments of maya glyphs 4. Saffron Walden, England.

1968 "Deciphering maya glyphs". Crambrook Institute of Science, News Letter, 37, 7. Blomfield Hills.

1968a "Deciphering maya hieroglyphs". Crambrook Institute of Science, News Letter, 87 (7): 82-87. Blomfield Hills.

1968b Prólogo a al obra: Tiempo y realidad en el pensamiento maya, de: Miguel León-Portilla. México. UNAM.

1969 "Maya hieroglyphs $\mathrm{Ku}$ as a value of the Cauac glyph". Congreso Internacional de Americanistas, 37, Mar del Plata, 1966. Buenos Aires.

1970 "The bacabs: their portraits and their glyphs". Monographs and papers in maya archaeology, Bullard Ed. Papers of the Peabody Museum of Archaelogy \& Ethnology, 61: 469-485. Cambridge, Mass.

1970a Maya history and religion. Norman, University of Oklahoma Press.

$1970 \mathrm{~b}$ "Sufijos numerales y medidas en yucateco". Estudios de Cultura Maya, 8: 319-340. México.

1972 A commentary on the Dresden maya Codex, a maya hieroglyph book. American Philosophical Society, Memoirs of the American Philosophical Society, 93. Philadelphia.

1972a Maya hieroglyphs without tears. London, Trustees of the British Museum.

1972b The maya of Belize: historical chapters since Columbus. [The Benex Press] Belize.

1972 c "Myth, methaphor and other factors in maya representations of fauna". Tulane University Simposia on the Art of America Latina. New Orleans. (mimeografiado).

1973 "Archaeological problems of the lowland maya; an analysis of the latest research in the highest centers of maya civilization, evaluating the positive knowledge and the gaps in information on that field". en The maya and their neighbors, p. 126-138. New York. (1a. Ed. 1940).

1973a "The painted capstone at Sacnite, Yucatan, and two other at Uxmal". Separata de: Indiana, p. 59-63. Berlín.

1974 "Maya astronomy". Sobretiro de Philosophical Transactions of the Royal Society of London Academy, 276: 83-98. London.

1974a "Canals" of the Candelaria basin, Campeche, Mexico". En Mesoamerican Archaeology, Hammond Ed. p. 297-302. Austin.

1975 Arqueología maya. México, Diana. (1a. Ed., 1965).

1975a Historia y religión de los mayas. Tr. Félix Blanco. México, S. XXI.

1975b Introducción a la obra: The hill-caves of Yucatan; a search for evidency of man's antiquity in the caverne of Central America, De Henry C. Mercer. Norman, University of Oklahoma Press.

En prensa "Nombres de días entre los mayas putunes". Estudios de Cultura Maya, 10, México. 


\section{NECROLOGIAS}

1935 "Berthold Laufer: 1874-1934". MAN. 35 (29). London.

1949 "Sylvanus Griswold Morley, 1883-1948". American Anthropologist, 51 (2): 293-297. Menasha.

1952 "Richard Charles Edward Long, 1872-1951". MAN, 52. London. (también en Boletín Bibliográfico de Antropología Americana, 14 (1): 300-302. México).

1953 "Enrique Juan Palacios, 1881-1953". American Antiquity, 19 (2): 152. Salt Lake City.

1960 "Juan Martínez Hernández, 1866-1959". American Antiquity, 25 (3) : 397-399. Salt Lake City.

1963 "Frans Blom, 1893-1963". Estudios de Cultura Maya, 3: 307-314. México.

1967 "Ralph Loveland Roys, 1879-1965". Estudios de Cultura Maya, 6: 421-431. México.

1973 "César Lizardi Ramos, 1895-1971". Estudios de Cultura Maya, 9: 381-391. México.

\section{RESENAS}

1932 "Eras of the thirteen gods and nine gods. Book of Chumayel, p. 4248". de: William E. Gates. Maya Society Quarterly, 2: 78-92. Baltimore, 1932. Maya Society Quarterly, 3: 120-122. Baltimore.

1933 En los confines de la selva lacandona; exploraciones en el Estado de Chiapas. De: Enrique Juan Palacios. México, 1928. Journal of Royal Geographic Society of London, 71: 555-556. London.

1933a Estudio arquitectónico de las ruinas mayas de Yucatán y Campeche. de: Federico E. Mariscal. México, 1928. Journal of Royal Geographic Society of London, 81: 555-556. London.

1933b People of the serpent. Life and adventures among the mayas. de: Edward H. Thompson. Boston, 1932. American Anthropologist, 35: 358-359. Menasha.

1938 Yucatan before and after the conquest; with other related documents, maps and illustrations. de: Fr. Diego de Landa. Tr. with notes by William Gates. Baltimore, 1937. American Anthropologist, 40: 309-310. Menasha.

1940 Codex Mendoza. The mexican manuscript known as the collection of Mendoza and preserved in the Bodleian Libray, Oxford. Ed. y Tr. James Cooper Clark. London, 1938. American Anthropologist, 42: 506-507. Menasha.

1941 Battlefield of the gods. Aspects of mexican history, art and exploration. De: Pal Kelemen. London, 1937. American Antiquity, 6 (3): 297-298. Salt Lake City.

1942 Aztecs of Mexico. Origin, rise and fall of the aztec nation. de: George C. Vaillant. New York, 1941. American Antiquity, 8 (1): 126. Salt Lake City.

1946 The origin and history of the mayas. De: Wells Jakeman M. Los Angeles, 1945. American Antiquity, 11 (3): 205-206. Salt Lake City.

1948 Historia tolteca-chichimeca. Liber in lingua nahuatl manuscriptus 
picturisque ornaments, ut est conservatus in Biblioteca Nationes Gallicae Parisiensi Sub Sumaris XLVI-LVIII bis, cum praefatione in lingua britannica, gallicia, germánica et hispana, at que indice paginarum. Ed. por Ernes Mengin. Havniae, 1942. American Anthropologist, 50: 110-112. Menasha.

1950 Popol Vuh the secred book of the ancient quiche maya. Tr. al inglés por Delia Goetz y Sylvanus G. Morley, del texto de Adrian Recinos. Norman, 1950. American Antiquity, 16(2): 175. Salt Lake City.

1951 The ancient maya. De: Sylvanus G. Morley. Stanford, 1946. American Journal of Archaeology, 55: 448-449. New York.

1951a The book of the jaguar priest. A translation of the book of Chilam Balam of Tizimin, Ed. with commentary by Maud Worcester Makemsom. New York, 1951. American Anthropoyogist, 53 (4): 546 547. Menasha.

1951b Florentine Codex. General history of the things of New Spain. Book 1: The gods. De: Fr. Bernardino de Sahagún. Tr. from aztec in to english with notes and illustrations by Arthur J. O. Anderson and Charles E. Dibble. Santa Fe, Calif., 1950. American Anthropoligist, 52: 272. Menasha.

1951c The four directions of time. An account of page one of Codex Féjerváry-Mayer. De: Cottie A. Burland. Santa Fe, Calif., 1950. Boletín Bibliográfico de Antropología Americana, 13 (2): 62-63. México.

1951d Temples in Yucatan. De: Laura Gilpin. New York, 1948. Boletín Bibliográfico de Antropología Americana, 13 (2): 76. México.

1952 Arte antiguo de México. De: Paul Westheim. México, 1950. Hispanic-American Historical Society Review, 32: 391-392. Durham, North Carolina.

1952a Guide to the Codex Perez. De: Ralph L. Roys. C.I.W., Contrib. 49, Washington, 1949. American Journal of Archaeology, 56 (1): 109110. New York.

1952b The prophecies for the maya tuns or years in the books of Chilam Balam of Tizimin and Mani. De: Ralph L. Roys. C.I.W., Contrib. 51, Washington, 1949. American Journal of Archaeology, 51 (1): 109-110. New York.

1952c The Tovar Calendar. De: George Kubler y Charles Gibson. Memoirs of the Conneticut Academy of Arts and Sciences. New Haven, Conn., 1951. American Journal of Archaeology, 56 (1): 110-111. New York.

1953 Florentine Codex. General history of the things of New Spain. Book 2: The ceremonies. De: Fr. Bernardino de Sahagún. Tr. from aztec in to english with notes and illustrations by Arthur J. O. Anderson and Charles E. Dibble. Santa Fe, Calif., 1951. American Anthropologist, 55: 251-252. Menasha.

1953a Drevjaja pis'mennost Central noj Ameriki. De: Yurij V. Knorozov Sovietskaja Etnografía, 3: 100-118. 1952. Yan, 2: 174-178. México.

1954 The "historical recolections". De: Gaspar Antonio Chi. Ed. and Tr. by M. Wells Jakeman. Provo, Utah, 1952. American Antiquity, 20 (2): 189. Salt Lake City. (Tlatoani, 5 (6): 91. México, 1952). 
1954a Florentine Codex. General history of the things of New Spain. Book 3: the origin of the gods. De: Fr. Bernardino de Sahagún. Tr. from aztec in to english with notes and illustrations by Arthur J. O. Anderson and Charles E. Dibble. Santa Fe, Calif., 1952. American Anthropologist, 56: 129-130. Menasha.

1954b Kurze formen-und begriff systematik der hierogylphen der mayahandschriften. De: Günter Zimmermann. Hamburg, 1953. American Antiquity, 20 (2): 189. Salt Lake City.

1955 The ruins of Zaculeo, Guatemala. De: Richard B. Woodbury and Aubrey S. Trik. 1953. Revista Interamericana de Bibliografía, 4 (4): 310-312. Washington.

1957 Altmexicanische kulturen. De: Walter Krickeberg. Berlin, 1956. The Hispanic-American Historical Society Review, 37: 497-498. Durham, North Carolina.

1957a La escultura del México antiguo. De: Paul Westheim. México, 1956. Revista Interamericana de Bibliografía, 7 (2): 167-168. Washington.

1958 The political geography of the Yucatan maya. De: Ralph L. Roys. C.I.W., Pub. 613, Washington, 1957. American Anthropologist, 60 (3): 591. Menasha.

1959 Chichen Itza and its cenote of sacrifice. A comparative study of contemporaneous maya and toltec. De: Alfred M. Tozzer. Memoirs of the Peabody Museum of Archaeology and Ethnology XI y XII, Cambridge, 1957. American Journal of Archaeology, 63: 119-120. New York.

1959a Die hieroglyphen der maya-handschriften. De: Günter Zimmermann. Hamburg, 1956. MAN, 59: 36. London.

1959b Mixtec e thnohistory. A method of analysis of the codical art. de: Philip Dark. Oxford University Press, 1958. MAN, 59: 35. London.

1960 Mexican manuscripts painting of the early colonial period. De: Donald Robertson. MAN, 60: 63. London.

1973 Edificios mayas Man Bauten der maya trazados en los años de 18861905 y descritos. De: Teobert Maler. Ed. póstuma a cargo de Gerdt Kuthcher. Berlín, 1971. Anales de Antropologa, UNAM, 10: 425. México. 